\title{
A NOTE ON THE IMPLICIT FUNCTION THEOREM FOR QUASI-LINEAR EIGENVALUE PROBLEMS
}

\author{
ROBIN NITTKA
}

\begin{abstract}
We consider the quasi-linear eigenvalue problem $-\Delta_{p} u=\lambda g(u)$ subject to Dirichlet boundary conditions on a bounded open set $\Omega$, where $g$ is a locally Lipschitz continuous functions. Imposing no further conditions on $\Omega$ or $g$ we show that for small $\lambda$ the problem has a bounded solution which is unique in the class of all small solutions. Moreover, this curve of solutions depends continuously on $\lambda$.
\end{abstract}

\section{IntRoduction}

We give an argument in the spirit of [CR73, §3] in order to prove existence of small solutions for the quasi-linear equation

$$
\left\{\begin{aligned}
-\Delta_{p} u & =\lambda g(u) & & \text { on } \Omega \\
u & =0 & & \text { on } \partial \Omega,
\end{aligned}\right.
$$

where $g: \mathbb{R} \rightarrow \mathbb{R}$ is locally Lipschitz continuous, $\lambda$ is small, $\Omega$ is an arbitrary bounded open subset of $\mathbb{R}^{N}$ and $p$ is in $\left(\frac{2 N}{N+2}, 2\right]$. More precisely, we show that there is a neighborhood $U$ of zero in $L^{\infty}(\Omega)$ such that for small $\lambda$ there is a unique solution $u_{\lambda} \in U \cap W_{0}^{1, p}(\Omega)$ of (1.1). Moreover, the dependence of $u_{\lambda}$ on $\lambda$ is Lipschitz continuous in the norms of $L^{\infty}(\Omega)$ and $W_{0}^{1, p}(\Omega)$.

Equation (1.1) is an example of a nonlinear eigenvalue problem (semilinear if $p=2$ and quasi-linear otherwise). If $g$ has subcritical growth, the situation is comparatively easy to handle. Namely, if $g(x) \leq(1+|x|)^{q}$ for all $x \in \mathbb{R}$ for some $q<\frac{N p}{N-p}-1$, then $u \mapsto g(u)$ is compact from $W_{0}^{1, p}(\Omega)$ to $W^{-1, p^{\prime}}(\Omega)$ and (1.1) can for example be attacked by fixed point arguments in the space $W_{0}^{1, p}(\Omega)$ of energy solutions. The critical case $q=\frac{N p}{N-p}-1$ is more difficult because $u \mapsto g(u)$ is no longer compact, but it is still possible to work in $W_{0}^{1, p}(\Omega)$. The subcritical and the critical case have been extensively studied, usually with variational methods under additional monotonicity assumptions. We refer to Ama76 for a detailed survey on semilinear eigenvalue problem and cite [DH01, KL03, AMdÓM05, CS07, ILU10] as examples for results in the quasi-linear case.

Here we also want to allow for supercritical growth. We think of $g=\exp$ as a model case. With this choice for $g$ equation (1.1) is often referred to as Gelfand's equation, in particular if $p=2$, and has a physical interpretation in astrophysics Cha57. For $p=2$ the situation is quite well understood, see for example [Dan88, CR75, AD07, PW02] for information about the number of solutions. For $p \neq 2$ less is known, but we refer to [AAP94] and references therein for results about existence, non-existence, multiplicity and stability. However, the methods of [AAP94] rely heavily on structure assumptions on $g$, namely some kind

Date: September 23, 2011.

2010 Mathematics Subject Classification. Primary: 35P30; Secondary: 47J07.

Key words and phrases. quasi-linear eigenvalue problem, Gelfand's equation, compact resolvent, implicit function theorem. 
of monotonicity and growth conditions, and thus do not generalize well. Here we propose a different approach to (1.1) based on the implicit function theorem, which goes along the lines of [CR75] where the case $p=2$ is studied. We emphasize the method's flexibility by allowing for general functions $g$ and by later on studying systems of $p$-Laplace equations instead of scalar equations.

There are two major difficulties to be overcome when using the implicit function theorem for (1.1). Firstly, one has to work in a space of bounded functions in order to be able to handle composition with $g$. For arbitrary domains a space of Hölder continuous functions like in [CR75] is not suitable, so we have to resort to $L^{\infty}(\Omega)$ like in Nit06]. Secondly, equation (1.1) does not behave well under linearization unless $p=2$, so we cannot expect that the implicit function theorem for continuously differentiable functions applies and have to use a topological version for compact Lipschitz maps. Thus we need compactness of the resolvent of the $p$ Laplace operator in $L^{\infty}(\Omega)$, which for rough domains seems to be a new result, and we need the local Lipschitz continuity of $\left(-\Delta_{p}\right)^{-1}$ in $L^{\infty}(\Omega)$, which has recently been obtained by Markus Biegert [Bie10]. Local Lipschitz continuity fails for $p>2$ and is not known for $p \in\left(1, \frac{2 N}{N+2}\right]$, which is the main reason why we have to restrict ourselves to $p \in\left(\frac{2 N}{N+2}, 2\right]$.

\section{2. $\Delta_{p}$ AND LOCAL LiPSCHITZ CONTINUity OF THE RESOlVENT}

Throughout the article $\Omega$ denotes a fixed bounded open subset of $\mathbb{R}^{N}$ and $p$ is a parameter satisfying $p>\frac{2 N}{N+2}$. Thus $W_{0}^{1, p}(\Omega)$ is compactly embedded into $L^{2}(\Omega)$. Later on we will also require that $p \leq 2$. We define

$$
\varphi_{p}(u):=\frac{1}{p} \int_{\Omega}|\nabla u|^{p}
$$

for $u \in W_{0}^{1, p}(\Omega) \subset L^{2}(\Omega)$ and $\varphi_{p}(u):=\infty$ for $u \in L^{2}(\Omega) \backslash W_{0}^{1, p}(\Omega)$. Then $\varphi_{p}$ is a proper, convex, lower semicontinuous functional on $L^{2}(\Omega)$. Its subgradient $-\Delta_{p}$ can be described by $u \in D\left(\Delta_{p}\right)$ and $-\Delta_{p} u=f \in W^{-1, p^{\prime}}(\Omega)$ if and only if $u \in W_{0}^{1, p}(\Omega)$ and

$$
\int_{\Omega}|\nabla u|^{p-2} \nabla u \nabla v=\langle f, v\rangle \text { for all } v \in W_{0}^{1, p}(\Omega),
$$

which means that on a formal level we can write $\Delta_{p} u=\operatorname{div}\left(|\nabla u|^{p-2} \nabla u\right)$. The operator $I-\alpha \Delta_{p}: W_{0}^{1, p}(\Omega) \rightarrow L^{2}(\Omega)$ is invertible for all $\alpha>0$. Its inverse $J_{\alpha}$ : $=\left(I+\alpha \Delta_{p}\right)^{-1}$ is contractive with respect to the norm of $L^{2}(\Omega)$. Moreover, the resolvent identity

$$
J_{\beta}=J_{\alpha}\left(\frac{\alpha}{\beta} I+\left(1-\frac{\alpha}{\beta}\right) J_{\beta}\right)
$$

holds for all $\alpha, \beta>0$. The operator $-\Delta_{p}$ is coercive on $L^{2}(\Omega)$ and hence invertible due to Poincaré's inequality. For proofs of these facts and further details we refer to Sho97.

We state the local Lipschitz continuity of $\left(-\Delta_{p}\right)^{-1}$ in the following lemma. This limit case is not included in Bie10, Theorem 5.1], but can be obtained from [Bie10, Theorem 3.1] by precisely the same arguments.

Lemma 2.1. Let $p \in\left(\frac{2 N}{N+2}, 2\right]$. If we pick $q \in(1, \infty)$ sufficiently large, we have the following property: if $u, v \in W_{0}^{1, p}(\Omega)$ satisfy $-\Delta_{p} u=f$ and $-\Delta_{p} v=g$ with functions $f, g \in L^{q}(\Omega)$. Then

$$
\|u-v\|_{\infty} \leq c\left(\|f\|_{q}+\|g\|_{q}\right)^{\frac{2-p}{p-1}}\|f-g\|_{q}
$$

for a constant $c$ that depends only on $p$ and $\Omega$. 
Lemma 2.1 says in particular that $\left(-\Delta_{p}\right)^{-1}$ is locally Lipschitz continuous on $L^{\infty}(\Omega)$. This seems to be a non-trivial result. For example, the scaling behavior

$$
\left(-\Delta_{p}\right)^{-1}(\lambda f)=\lambda^{\frac{1}{p-1}}\left(-\Delta_{p}\right)^{-1} f .
$$

implies that $\left(-\Delta_{p}\right)^{-1}$ cannot be Lipschitz continuous in any neighborhood of zero if $p>2$, and local Lipschitz continuity for $p \in\left(1, \frac{2 N}{N+2}\right]$ seems to be an open problem.

On the other hand, global Lipschitz continuity of $J_{\alpha}:=\left(I-\alpha \Delta_{p}\right)^{-1}$ on $L^{\infty}(\Omega)$ for every $\alpha>0$ and every $p \in(1, \infty)$ is comparatively easy to establish.

Lemma 2.2. For every $q \in[2, \infty]$ the restriction of $J_{\alpha}$ to $L^{q}(\Omega)$ is contractive in the norm of $L^{q}(\Omega)$.

Proof. It can be proved as in [CG03, Theorem 4.1] that $\varphi_{p}$ defined in (2.1) is a nonlinear Dirichlet form in the sense of [CG03], i.e., the corresponding semigroup on $L^{2}(\Omega)$ is order preserving and contractive in the norm of $L^{\infty}(\Omega)$. Thus the resolvent has the same properties [Bré73], and by interpolation we obtain the result for all $q \in[2, \infty]$, see for example [Bro69].

\section{Compact Resolvent}

We prove that $J_{\alpha}:=\left(I-\alpha \Delta_{p}\right)^{-1}$ acts as a compact operator on $L^{\infty}(\Omega)$. Recall that a nonlinear operator is called compact if it is continuous and maps bounded sets to relatively compact sets. An operator is called bounded if it maps bounded sets to bounded sets, so trivially every compact operator is bounded. Compactness of $J_{\alpha}$ (and similar operators) in $L^{q}$-spaces for $q<\infty$ has been studied in DD09, $\S 5]$. However, the case $q=\infty$ seems to be new and is in fact not accessible by the methods in [DD09]. To be more precise, the argument in DD09] goes via an interpolation inequality, so in order to transfer their idea to $L^{\infty}(\Omega)$ one needs to prove boundedness of the resolvent into in a space of higher regularity. This regularity, however, is only to be expected if $\Omega$ is sufficiently smooth. Thus instead we use the following different approach, adapted from [AD07, Theorem 7.1], which we present in an abstract framework.

Definition 3.1. Let $X$ be a Banach space. We say that a family $\left(J_{\alpha}\right)_{\alpha>0}$ of (possibly nonlinear) operators on $X$ is a (nonlinear) pseudo-resolvent if (2.2) holds for all $\alpha, \beta>0$.

Lemma 3.2. Let $\left(J_{\alpha}\right)_{\alpha>0}$ be a pseudo-resolvent on a Banach space $X$. Assume that the family $\left(J_{\alpha}\right)_{\alpha>0}$ is uniformly bounded and that $\left(J_{\alpha}\right)_{\alpha>0}$ is equicontinuous on bounded subsets of $X$. Then for all $k \in \mathbb{N}_{0}$ and all $\beta>0$ we have $J_{\alpha}^{k} J_{\beta} \rightarrow J_{\beta}$ as $\alpha \rightarrow 0$ uniformly on bounded sets.

Proof. Let $\beta>0$ be fixed. We prove the claim by induction. For $k=0$ there is nothing to show. So assume that $J_{\alpha}^{k} J_{\beta} \rightarrow J_{\beta}$ as $\alpha \rightarrow 0$ uniformly on bounded sets for some $k \in \mathbb{N}$. By (2.2),

$$
J_{\alpha}^{k} J_{\beta}=J_{\alpha}^{k+1}\left(\frac{\alpha}{\beta} I+\left(1-\frac{\alpha}{\beta}\right) J_{\beta}\right)=J_{\alpha}^{k+1} J_{\beta}+T_{\alpha}
$$

with

$$
T_{\alpha}:=J_{\alpha}^{k+1}\left(\frac{\alpha}{\beta} I+\left(1-\frac{\alpha}{\beta}\right) J_{\beta}\right)-J_{\alpha}^{k+1} J_{\beta} .
$$

Hence it suffices to show that $T_{\alpha} \rightarrow 0$ uniformly on bounded sets. To this end let $\left(x_{\alpha}\right)_{\alpha>0}$ be a bounded family in $X$. Since $J_{\beta}$ is bounded, there exists $R>0$ such that the vectors

$$
y_{\alpha}:=\frac{\alpha}{\beta} x_{\alpha}+\left(1-\frac{\alpha}{\beta}\right) J_{\beta} x_{\alpha} \quad \text { and } \quad z_{\alpha}:=J_{\beta} x_{\alpha}
$$


lie in the ball $B(0, R)$ for every $\alpha<1$. In particular,

$$
\left\|y_{\alpha}-z_{\alpha}\right\|=\frac{\alpha}{\beta}\left\|x_{\alpha}-J_{\beta} x_{\alpha}\right\| \rightarrow 0 \quad(\alpha \rightarrow 0) .
$$

By uniform equicontinuity this implies that $\left\|J_{\alpha} y_{\alpha}-J_{\alpha} z_{\alpha}\right\| \rightarrow 0$ as $\alpha \rightarrow 0$. Since also the families $\left(J_{\alpha} y_{\alpha}\right)_{\alpha>0}$ and $\left(J_{\alpha} z_{\alpha}\right)_{\alpha>0}$ are bounded, we can proceed by induction and deduce that

$$
\left\|T_{\alpha} x_{\alpha}\right\|=\left\|J_{\alpha}^{k+1} y_{\alpha}-J_{\alpha}^{k+1} z_{\alpha}\right\| \rightarrow 0 \quad(\alpha \rightarrow 0) .
$$

Thus $T_{\alpha} \rightarrow 0$ as $\alpha \rightarrow 0$ uniformly on bounded sets.

Theorem 3.3. Let $X$ and $Y$ be a Banach spaces with $Y$ continuously embedded into $X$. Let $\left(J_{\alpha}\right)_{\alpha>0}$ be a pseudo-resolvent on $X$ consisting of continuous, bounded operators. Assume that $J_{\alpha}$ is compact on $X$ for one (or, equivalently, for all) $\alpha>0$. Assume moreover that $J_{\alpha}(Y) \subset Y$ for all $\alpha>0$ and that $\left(\left.J_{\alpha}\right|_{Y}\right)_{\alpha>0}$ is uniformly bounded and uniformly equicontinuous on bounded subsets of $Y$. Finally, assume that there exist $k \in \mathbb{N}$ and $\beta>0$ such that $J_{\alpha}^{k}$ is bounded and continuous from $X$ into $Y$ for all $\alpha>0$. Then $\left.J_{\alpha}\right|_{Y}$ is compact on $Y$ for every $\alpha>0$.

Proof. Since $J_{\alpha}^{k}$ is bounded from $X$ to $Y$ and $J_{\beta}$ is compact on $X$, the operator $J_{\alpha}^{k} J_{\beta}$ is a compact operator from $X$ to $Y$ for all $\alpha>0$ and $\beta>0$. Hence $\left.J_{\alpha}^{k} J_{\beta}\right|_{Y}$ is a compact operator on $Y$. From Lemma 3.2 we obtain that $\left.\left.J_{\alpha}^{k} J_{\beta}\right|_{Y} \rightarrow J_{\beta}\right|_{Y}$ as $\alpha \rightarrow 0$ uniformly on bounded sets. Hence $\left.J_{\beta}\right|_{Y}$ is compact.

We need Theorem 3.3 only in the following situation.

Example 3.4. Let $\Omega$ be an open, bounded subset of $\mathbb{R}^{N}$. For every $p \in\left(\frac{2 N}{N+2}, \infty\right)$ the operator $-\Delta_{p}$ has compact resolvent on $L^{\infty}(\Omega)$. Moreover, also the operator $\left(-\Delta_{p}\right)^{-1}$ is compact on $L^{\infty}(\Omega)$.

Proof. Define $J_{\alpha}:=\left(I-\alpha \Delta_{p}\right)^{-1}$. Since $u \mapsto \varphi_{p}\left(J_{\alpha} u\right)$ is bounded on bounded subsets of $L^{2}(\Omega)$, see for example [Sho97, Proposition IV.1.8], the operator $J_{\alpha}$ is bounded from $L^{2}(\Omega)$ to $W_{0}^{1, p}(\Omega)$. Since in addition $J_{\alpha}$ is continuous (in fact even contractive) on $L^{2}(\Omega)$, it is compact on $L^{2}(\Omega)$.

The restriction of $J_{\alpha}$ to $L^{\infty}(\Omega)$ is equicontinuous and uniformly bounded by Lemma 2.2. Iterating the estimates in [DD09, Theorem 2.5] we obtain that $J_{\alpha}^{k}$ is bounded from $L^{2}(\Omega)$ to $L^{\infty}(\Omega)$ for some $k \in \mathbb{N}$. Hence $J_{\alpha}^{k}$ is continuous from $L^{2}(\Omega)$ to $L^{q}(\Omega)$ for every $q<\infty$ by interpolation. Thus $J_{\alpha}^{k+1}$ is bounded and continuous from $L^{2}(\Omega)$ to $L^{\infty}(\Omega)$ by Lemma 2.1. Compactness of $J_{\alpha}$ on $L^{\infty}(\Omega)$ now follows from Theorem 3.3 .

Finally, $\left(-\Delta_{p}\right)^{-1}$ is bounded and continuous on $L^{\infty}(\Omega)$ by Lemma 2.1 Hence the trivial identity

$$
\left(-\Delta_{p}\right)^{-1}=J_{\alpha}\left(\left(-\Delta_{p}\right)^{-1}+\alpha I\right)
$$

which is valid for all $\alpha>0$, shows that $\left(-\Delta_{p}\right)^{-1}$ is compact on $L^{\infty}(\Omega)$.

\section{A topologicAl IMPLiCit FUNCTION THEOREM}

We need an implicit function theorem in Banach spaces for functions that are merely Lipschitz continuous. The following argument is based on a rather deep theorem about local inverses that relies on topological degree theory. For finitedimensional spaces this idea has been described in [Wue08]. The generalization to infinite dimensions is straight-forward, but seems not to be widely known.

Proposition 4.1. Let $X, Y$ and $Z$ be Banach spaces. Let $K: X \times Y \rightarrow Z$ be a locally Lipschitz continuous compact operator. Assume that $K(0,0)=0$ and that there exist $\kappa<1$ and $\delta>0$ such that $\left\|K\left(x, y_{1}\right)-K\left(x, y_{2}\right)\right\| \leq \kappa\left\|y_{1}-y_{2}\right\|$ whenever $x \in B_{X}(0, \delta)$ and $y_{1}, y_{2} \in B_{Y}(0, \delta)$. Then there exists a neighborhood $U \times V$ of 
$(0,0)$ in $X \times Y$ and a Lipschitz continuous function $\varphi: U \rightarrow V$ with the property that for $(x, y) \in U \times V$ we have $K(x, y)=y$ if and only if $y=\varphi(x)$.

Proof. Let $L \geq 0$ denote the Lipschitz constant of $K$ in a neighborhood of $(0,0)$ and pick $0<\varepsilon<L^{-1}$. Setting $f(x, y):=(x, \varepsilon y-\varepsilon K(x, y))$ we easily obtain that

$$
\left\|f\left(x_{1}, y_{1}\right)-f\left(x_{2}, y_{2}\right)\right\| \geq(1-\varepsilon L)\left\|x_{1}-x_{2}\right\|+\varepsilon(1-\kappa)\left\|y_{1}-y_{2}\right\|,
$$

from which we see that $f$ is injective near $(0,0)$. Since $f$ is a compact perturbation of the identity this implies that $f$ is continuously invertible in a neighborhood of $(0,0)$, see [Ber77, (5.4.11)]. Define $\varphi(x)$ to be the second component of $f^{-1}(x, 0)$. By (4.1) the operator $f^{-1}$ is Lipschitz continuous, hence so is $\varphi$. Moreover, $K(x, y)=y$ if and only if $f(x, y)=(x, 0)$, which for $(x, y)$ in a neighborhood of $(0,0)$ is equivalent to $\varphi(x)=y$.

\section{Existence OF SMALL SOLUTiOnS}

We now prove existence of small solutions of (1.1) (or the more general equation (5.1) ) by constructing a curve of solutions emanating from $(\lambda, u)=(0,0)$, for which we use the implicit function theorem of the previous section. In order to emphasize the flexibility of our approach, in particular that the method does not rely on the variational structure of (1.1), we consider a quasi-linear reaction-diffusion system instead of a scalar equation, compare also [AC02].

Theorem 5.1. Let $\Omega \subset \mathbb{R}^{N}$ be open and bounded, let $d \in \mathbb{N}$, fix numbers $p_{1}, \ldots, p_{d} \in\left(\frac{2 N}{N+2}, 2\right]$ and let $g: \mathbb{R}^{d} \rightarrow \mathbb{R}^{d}$ be locally Lipschitz continuous. Then there exist $\lambda_{0}>0$ such that for every $\lambda \in\left(-\lambda_{0}, \lambda_{0}\right)$ the system

$$
\left\{\begin{aligned}
-\Delta_{p_{i}} u_{i} & =\lambda g_{i}\left(u_{1}, \ldots, u_{d}\right) & & \text { on } \Omega \\
u_{i} & =0 & & \text { on } \partial \Omega
\end{aligned}\right.
$$

has a solution $\left(u_{1}, \ldots, u_{d}\right)=u=u_{\lambda}$ in $W_{0}^{1, p}\left(\Omega ; \mathbb{R}^{d}\right) \cap L^{\infty}\left(\Omega ; \mathbb{R}^{d}\right)$; in particular $g\left(u_{\lambda}\right) \in L^{\infty}\left(\Omega ; \mathbb{R}^{d}\right) \subset W^{-1, p^{\prime}}\left(\Omega ; \mathbb{R}^{d}\right)$ so that the notion of an (energy) solution applies here.

Moreover, the mapping $\lambda \rightarrow u_{\lambda}$ is Lipschitz continuous from $\left(-\lambda_{0}, \lambda_{0}\right)$ to $L^{\infty}(\Omega) \cap$ $W_{0}^{1, p}(\Omega)$. Finally, there exist $\varepsilon>0$ and $\lambda_{1} \in\left(0, \lambda_{0}\right)$ such that for all $\lambda \in\left(-\lambda_{1}, \lambda_{1}\right)$ the function $u_{\lambda}$ is the only solution $u$ of (5.1) that satisfies $\|u\|_{\infty} \leq \varepsilon$.

Proof. Consider the nonlinear operator $K$ from $\mathbb{R} \times L^{\infty}\left(\Omega ; \mathbb{R}^{d}\right)$ to $L^{\infty}\left(\Omega ; \mathbb{R}^{d}\right)$ given by

$$
(K(\lambda, u))_{i}:=\left(-\Delta_{p_{i}}\right)^{-1}\left(\lambda g_{i}(u)\right) .
$$

By Example 3.4 the operator $K$ is compact. By Lemma 2.1 and local Lipschitz continuity of $g$ the operator $K$ is locally Lipschitz continuous. Moreover,

$$
\begin{aligned}
\|K(\lambda, u)-K(\lambda, \tilde{u})\|_{\infty} & \leq c_{1} \lambda^{\frac{1}{p-1}}\left(\|g(u)\|_{\infty}+\|g(\tilde{u})\|_{\infty}\right)\|g(u)-g(\tilde{u})\|_{\infty} \\
& \leq c_{2} \lambda^{\frac{1}{p-1}}\|u-\tilde{u}\|_{\infty}
\end{aligned}
$$

by Lemma 2.1 with constants $c_{1}$ and $c_{2}$ that depend only on the $p_{i}$, an upper bound $R$ for $u$ and $\tilde{u}$ in $L^{\infty}\left(\Omega ; \mathbb{R}^{d}\right)$ and the Lipschitz constant of $g$ on $B_{\mathbb{R}^{d}}(0, R)$.

Hence the assumptions of Proposition 4.1 are satisfied. We deduce that the equation $K(\lambda, u)=u$, i.e., problem (5.1), is locally solved by an implicit function $u_{\lambda}:=\varphi(\lambda)$, that there are no other $L^{\infty}$-small solutions for small $\lambda$ and that the dependence of $u_{\lambda}$ on $\lambda$ is Lipschitz continuous with respect to the norm of $L^{\infty}(\Omega)$. Lipschitz continuous dependence in the norm of $W_{0}^{1, p}(\Omega)$ follows from the identity $u_{\lambda}=\left(-\Delta_{p}\right)^{-1}\left(\lambda g\left(u_{\lambda}\right)\right)$ since $\left(-\Delta_{p}\right)^{-1}$ is locally Lipschitz continuous from $W^{-1, p^{\prime}}(\Omega)$ to $W_{0}^{1, p}(\Omega)$, see [Nit10, Theorem 3.3.18 and Example 3.3.23]. 
We have restricted ourselves to Dirichlet boundary conditions for simplicity. Still, essentially the same arguments apply to the system

$$
\left\{\begin{array}{clrl}
\left|u_{i}\right|^{p_{i}-2} u_{i}-\Delta_{p_{i}} u_{i} & =\lambda g_{i}\left(u_{1}, \ldots, u_{d}\right) & & \text { on } \Omega \\
\left|\nabla u_{i}\right|^{p-2} \frac{\partial u_{i}}{\partial \nu} & =0 & & \text { on } \partial \Omega
\end{array}\right.
$$

subject to Neumann boundary conditions if $\Omega \subset \mathbb{R}^{N}$ is a bounded Lipschitz domain. Moreover, we could let $g$ depend on $x \in \Omega$ or consider more general quasi-linear operators for which the assumptions of [Bie10, Theorem 3.1] hold.

Remark 5.2. Theorem 5.1 generalizes the first step of the argument in [CR75] from $p=2$ to a larger range. It would now be interesting to study the behavior of $u_{\lambda}$ as $\lambda$ increases. For $d=1$ and $g=\exp$, i.e., Gelfand's equation with arbitrary $p \in(1, \infty)$, it is known that there are no solutions for sufficiently large $\lambda$, see AAP94. One would suspect that one observes the same turning point structure as described in [CR75 for $p=2$ where the curve ceases to exist. In fact, this is true if $\Omega$ is a ball [JS02, Example 3.1], but it is not obvious how to handle the case $p \neq 2$ for general domains.

\section{REFERENCES}

[AAP94] J. Garcia Azorero, I. Peral Alonso, and J.P. Puel, Quasilinear problems with exponential growth in the reaction term, Nonlinear Analysis: Theory, Methods \& Applications 22 (1994), no. 4, 481-498.

[AC02] Céline Azizieh and Philippe Clément, A priori estimates and continuation methods for positive solutions of p-Laplace equations, J. Differential Equations 179 (2002), no. 1, 213-245.

[AD07] Wolfgang Arendt and Daniel Daners, Uniform convergence for elliptic problems on varying domains, Math. Nachr. 280 (2007), no. 1-2, 28 49.

[Ama76] Herbert Amann, Fixed point equations and nonlinear eigenvalue problems in ordered Banach spaces, SIAM Rev. 18 (1976), no. 4, 620-709.

[AMdÓM05] Emerson A. M. Abreu, João Marcos do Ó, and Everaldo S. Medeiros, Multiplicity of positive solutions for a class of quasilinear nonhomogeneous Neumann problems, Nonlinear Anal. 60 (2005), no. 8, 14431471.

[Ber77] Melvin S. Berger, Nonlinearity and functional analysis, Academic Press [Harcourt Brace Jovanovich Publishers], New York, 1977, Lectures on nonlinear problems in mathematical analysis, Pure and Applied Mathematics.

[Bie10] Markus Biegert, A priori estimates for the difference of solutions to quasi-linear elliptic equations, Manuscripta Math. 133 (2010), no. 3-4, 273-306.

[Bré73] H. Brézis, Opérateurs maximaux monotones et semi-groupes de contractions dans les espaces de Hilbert, North-Holland Publishing Co., Amsterdam, 1973, North-Holland Mathematics Studies, No. 5. Notas de Matemática (50).

[Bro69] Félix E. Browder, Remarks on nonlinear interpolation in Banach spaces, J. Functional Analysis 4 (1969), 390-403.

[CG03] Fabio Cipriani and Gabriele Grillo, Nonlinear Markov semigroups, nonlinear Dirichlet forms and applications to minimal surfaces, J. Reine Angew. Math. 562 (2003), 201-235. 
[Cha57] S. Chandrasekhar, An introduction to the study of stellar structure, Dover Publications Inc., New York, N. Y., 1957. MR 0092663 $(19,1142 \mathrm{~b})$

[CR73] Michael G. Crandall and Paul H. Rabinowitz, Bifurcation, perturbation of simple eigenvalues and linearized stability, Arch. Rational Mech. Anal. 52 (1973), 161-180.

[CR75] _ Some continuation and variational methods for positive solutions of nonlinear elliptic eigenvalue problems, Arch. Rational Mech. Anal. 58 (1975), no. 3, 207-218.

[CS07] Xavier Cabré and Manel Sanchón, Semi-stable and extremal solutions of reaction equations involving the p-Laplacian, Commun. Pure Appl. Anal. 6 (2007), no. 1, 43-67.

[Dan88] E. N. Dancer, The effect of domain shape on the number of positive solutions of certain nonlinear equations, J. Differential Equations $\mathbf{7 4}$ (1988), no. 1, 120-156.

[DD09] Daniel Daners and Pavel Drábek, A priori estimates for a class of quasi-linear elliptic equations, Trans. Amer. Math. Soc. 361 (2009), no. 12, 6475-6500.

[DH01] Pavel Drábek and Jesús Hernández, Existence and uniqueness of positive solutions for some quasilinear elliptic problems, Nonlinear Anal. 44 (2001), no. 2, Ser. A: Theory Methods, 189-204.

[ILU10] Leonelo Iturriaga, Sebastián Lorca, and Pedro Ubilla, A quasilinear problem without the Ambrosetti-Rabinowitz-type condition, Proc. Roy. Soc. Edinburgh Sect. A 140 (2010), no. 2, 391-398.

[JS02] Jon Jacobsen and Klaus Schmitt, The Liouville-Bratu-Gelfand problem for radial operators, J. Differential Equations 184 (2002), no. 1, 283-298.

[KL03] Dimitrios A. Kandilakis and Athanasios N. Lyberopoulos, Multiplicity of positive solutions for some quasilinear Dirichlet problems on bounded domains in $\mathbb{R}^{n}$, Comment. Math. Univ. Carolin. 44 (2003), no. 4, 645-658.

[Nit06] R. Nittka, The number of solutions of non-linear elliptic equations on varying domains, Master's thesis, University of Ulm, 2006.

[Nit10] _ Elliptic and parabolic problems with Robin boundary conditions on Lipschitz domains, Ph.D. thesis, University of Ulm, 2010.

[PW02] Michael Plum and Christian Wieners, New solutions of the Gelfand problem, J. Math. Anal. Appl. 269 (2002), no. 2, 588-606.

[Sho97] R. E. Showalter, Monotone operators in Banach space and nonlinear partial differential equations, Mathematical Surveys and Monographs, vol. 49, American Mathematical Society, Providence, RI, 1997.

[Wue08] M. Wuertz, The implicit function theorem for Lipschitz functions and applications, Master's thesis, University of Missouri, 2008.

Robin Nittka, Max Planck Institute for Mathematics in the Sciences, Inselstr. 22, 04103 Leipzig, Germany

E-mail address: nittka@mis.mpg.de 